\title{
Études/Inuit/Studies
}

\section{Soutenir la sécurité alimentaire dans le Grand Nord : projets communautaires d'agriculture sous serre au Nunavik et au Nunavut \author{
greenhouse projects in Nunavik and Nunavut
} \\ Supporting food security in the Far North: Community}

\author{
Annie Lamalice, Ellen Avard, Véronique Coxam, Thora Herrmann, Caroline \\ Desbiens, Yohann Wittrant et Sylvie Blangy
}

Volume 40, numéro 1, 2016

La santé des Inuit

Inuit health

URI : https://id.erudit.org/iderudit/1040149ar

DOI : https://doi.org/10.7202/1040149ar

Aller au sommaire du numéro

Éditeur(s)

Association Inuksiutiit Katimajiit Inc.

Centre interuniversitaire d'études et de recherches autochtones (CIÉRA)

ISSN

0701-1008 (imprimé)

1708-5268 (numérique)

Découvrir la revue

Citer cet article

Lamalice, A., Avard, E., Coxam, V., Herrmann, T., Desbiens, C., Wittrant, Y. \& Blangy, S. (2016). Soutenir la sécurité alimentaire dans le Grand Nord : projets communautaires d'agriculture sous serre au Nunavik et au Nunavut.

Études/Inuit/Studies, 40(1), 147-169. https://doi.org/10.7202/1040149ar

\section{Résumé de l'article}

Face aux grands défis de la sécurité alimentaire dans les villages du Nunavik et du Nunavut, le développement d'une agriculture nordique est envisagé comme une solution innovante. Visant la santé et le bien-être des communautés inuit, l'implantation d'une serre communautaire permet d'accroître l'offre en produits frais locaux et d'améliorer la qualité de l'alimentation, dans une démarche durable tenant compte de la dimension culturelle de la sécurité alimentaire. Cet article décrit les projets de serres communautaires de Kuujjuaq (Nunavik) et d'Iqaluit (Nunavut), ainsi que l'actuelle étude de faisabilité d'un projet de serre communautaire à Kangiqsujuaq (Nunavik). Nous présentons d'abord notre méthodologie qui repose sur les principes de la recherche participative basée dans la communauté. Nous nous penchons ensuite sur les principaux axes du projet de recherche : la contribution d'un projet de serre à la sécurité et à la souveraineté alimentaires et les défis techniques et organisationnels à relever pour l'optimisation d'une serre en contexte nordique. La démarche employée dans ce projet interdisciplinaire permet de construire, avec la communauté, un système d'approvisionnement local et durable et de comprendre la contribution d'un projet horticole à l'amélioration de la qualité de vie et de la santé des habitants. 


\title{
Soutenir la sécurité alimentaire dans le Grand Nord: projets communautaires d'agriculture sous serre au Nunavik et au Nunavut
}

\author{
Annie Lamalice', Ellen Avardii, Véronique Coxamiii, \\ Thora Herrmanniv, Caroline Desbiensv, Yohann Wittrantvi, \\ Sylvie Blangyvii
}

\begin{abstract}
Supporting food security in the Far North: Community greenhouse projects in Nunavik and Nunavut

Development of northern agriculture is seen as an innovative solution to the great challenges of food security in the villages of Nunavik and Nunavut. Establishing a community greenhouse can improve the health and well-being of Inuit communities by sustainably increasing the supply of fresh local produce and by improving food quality while not ignoring the cultural dimension of food security. This article describes community greenhouse projects in Kuujjuaq (Nunavik) and lqaluit (Nunavut), and a current study on the feasibility of a community greenhouse project in Kangiqsujuaq (Nunavik). We first present our methodology which follows the principles of
\end{abstract}

i Centre d'Écologie fonctionnelle et évolutive UMR 5175, CNRS, Université de Montpellier, France. Adresse postale: Département de géographie, Université de Montréal, Pavillon 520, chemin de la Côte-Sainte-Catherine, C.P. 6128, succursale Centre-Ville, Montréal, Québec H3C 3J7, Canada. annie.lamalice@umontreal.ca.

ii Nunavik Research Centre, Makivik Corporation, P.O. Box 179, Kuujjuaq, Québec J0M 1C0, Canada.e_avard@makivik.org.

iii Unité de Nutrition humaine UMR 1019, Équipe Alimentation, squelette et métabolismes, Université Clermont Auvergne, 63122 Saint-Genès-Champanelle, France. Veronique.Coxam@ clermont.inra.fr.

iv Département de géographie, Université de Montréal, C.P. 6128, succursale Centre-Ville, Montréal, Québec H3C 3J7, Canada. thora.martina.herrmann@umontreal.ca.

v Département de géographie, Université Laval, Pavillon Abitibi-Price, local 3185, 2405 rue de la Terrasse, Québec, Québec G1V 0A6, Canada. caroline.desbiens@ggr.ulaval.ca.

vi Unité de Nutrition humaine UMR 1019, Équipe Alimentation, squelette et métabolismes, Université Clermont Auvergne, 63122 Saint-Genès-Champanelle, France. yohann.wittrant@ clermont.inra.fr.

vii Centre d'Écologie fonctionnelle et évolutive UMR 5175, CNRS - Université de Montpellier Université Paul Valéry Montpellier - EPHE, 1919 route de Mende, 34293 Montpellier, France. Sylvie.blangy@cefe.cnrs.fr. 
community-based participatory research. We then turn to the main avenues of our research: the contribution of a greenhouse project to food security and sovereignty and the technical and organizational challenges of optimizing a greenhouse in a northern setting. Through this interdisciplinary project, it is possible to work with the community to build a local and sustainable food supply system and learn how a horticultural project can improve the quality of life and health of its inhabitants.

\section{RÉSUMÉ}

Soutenir la sécurité alimentaire dans le Grand Nord: projets communautaires d'agriculture sous serre au Nunavik et au Nunavut

Face aux grands défis de la sécurité alimentaire dans les villages du Nunavik et du Nunavut, le développement d'une agriculture nordique est envisagé comme une solution innovante. Visant la santé et le bien-être des communautés inuit, l'implantation d'une serre communautaire permet d'accroître I'offre en produits frais locaux et d'améliorer la qualité de l'alimentation, dans une démarche durable tenant compte de la dimension culturelle de la sécurité alimentaire. Cet article décrit les projets de serres communautaires de Kuujjuaq (Nunavik) et d'Iqaluit (Nunavut), ainsi que l'actuelle étude de faisabilité d'un projet de serre communautaire à Kangiqsujuaq (Nunavik). Nous présentons d'abord notre méthodologie qui repose sur les principes de la recherche participative basée dans la communauté. Nous nous penchons ensuite sur les principaux axes du projet de recherche: la contribution d'un projet de serre à la sécurité et à la souveraineté alimentaires et les défis techniques et organisationnels à relever pour l'optimisation d'une serre en contexte nordique. La démarche employée dans ce projet interdisciplinaire permet de construire, avec la communauté, un système d'approvisionnement local et durable et de comprendre la contribution d'un projet horticole à l'amélioration de la qualité de vie et de la santé des habitants.

\section{Introduction}

L'ouverture d'épiceries dans les villages du Nunavik, même si elle a pu concourir à éviter les pénuries saisonnières (Avard 2015), a engendré une transformation du système alimentaire et une transition nutritionnelle rapides et drastiques. Cette occidentalisation du régime alimentaire a fait reculer la contribution des aliments traditionnels à l'apport énergétique et nutritionnel au profit des produits achetés en épicerie, en particulier chez les jeunes (Counil et al. 2009, 2011). À titre d'exemple, en 2004 au Nunavik, 99\% des glucides provenaient des produits importés tandis que seulement $40 \%$ des apports en protéines et $18 \%$ des apports en lipides étaient issus des aliments du terroir (Blanchet et Rochette 2008). Les aliments du marché, de mauvaise qualité, riches en sucres et en gras, tels que boissons gazeuses et croustilles, représentaient alors $36 \%$ de l'apport énergétique moyen (ibid.). Ce nouveau régime alimentaire a contribué à l'émergence de maladies cardiovasculaires, d'obésité et de diabète de type 2 (Château-Degat 2011; Damman et al. 2008). Par conséquent, la sécurité alimentaire, qui est atteinte lorsque "tous les êtres humains ont, à tout moment, la possibilité physique, sociale et économique de se procurer une nourriture suffisante, saine et nutritive leur permettant de satisfaire leurs besoins et 
préférences alimentaires pour mener une vie saine et active» (FAO 2006), demeure à de nombreux égards absente au Nunavik et au Nunavut.

Malgré plusieurs décennies d'efforts gouvernementaux, l'insécurité alimentaire demeure un problème aussi important que complexe dans l'Arctique canadien. Cette insécurité concerne à la fois la quantité et la qualité des aliments consommés et s'explique par différents facteurs: le coût de la vie très élevé, l'accès de plus en plus limité aux produits issus des activités traditionnelles de subsistance (pêche, chasse, cueillette), une méconnaissance des bienfaits et méfaits des aliments du marché, ainsi que l'impact des changements climatiques et de la pollution de l'environnement sur les circuits alimentaires traditionnels (Holzman 2011; Huet et al. 2012; Power 2008). Comparativement aux pratiques actuelles, les aliments traditionnels sont significativement $(p<0,01)$ moins riches en graisses et sucres et plus concentrés en protéines, vitamines (A, D, E, riboflavine, B6), minéraux et oligo-éléments (fer, zinc, cuivre, magnésium, manganèse, phosphore, potassium et sélénium) (Kuhnlein et al. 2004; Sharma et al. 2010). En outre, les acides gras apportés par les produits du terroir sont d'excellente qualité en acides eicosapentaénoïque et docosahexaénoïque (Blanchet et al. 2000), dont l'intérêt pour la santé est largement décrit dans la littérature scientifique (Abedi et Sahari 2014; Sperling et Nelson 2016). Les recommandations actuelles sont de maintenir ou d'augmenter la consommation d'aliments du terroir provenant de la chasse, de la pêche ou de la cueillette, car ils contribuent au bien-être physique et psychologique des Inuit, et de réduire la consommation d'aliments achetés à l'épicerie, qui sont de faible qualité nutritionnelle, au profit de celle d'aliments de bonne valeur nutritive (tels que les fruits et les légumes, les grains entiers [céréales et légumineuses] et les produits laitiers) (Blanchet et Rochette 2008). Cependant, ces derniers sont difficilement accessibles car leur acheminement par avion tend à en altérer la qualité et surtout à en faire grimper le prix (Huet et al. 2012). En somme, la sécurité alimentaire globale s'améliorerait avec un meilleur accès aux aliments de bonne qualité du marché (Chan et al. 2006; Ford 2009) et c'est l'un des objectifs qui doit être ciblé en priorité.

Le présent article s'intéresse à deux projets de recherche visant à développer et instaurer des solutions alternatives adaptées à la culture de ces régions nordiques éloignées. Plus précisément, il s'intéresse à la sécurité alimentaire en proposant la mise en ouvre de projets horticoles innovants permettant un approvisionnement en produits frais, localement disponibles et à coût réduit. Dans les milieux nordiques éloignés des grands centres, l'innovation sera la clé de l'adaptation et de la résilience à une époque de changements socioculturels et environnementaux très rapides (Avard 2015). L'objectif principal de cette démarche est d'améliorer le bien-être et la santé de la population inuit en explorant le potentiel de l'agriculture circumpolaire pour la construction d'une nouvelle stratégie alimentaire qui soit durable et adaptée culturellement. Trouver des moyens d'adaptation qui répondent aux besoins locaux et qui soient compatibles avec les normes culturelles est un défi majeur pour les populations 
inuit du Canada (Pearce et al. 2015) et nous soutenons que les projets de serres peuvent compter parmi ces moyens.

Nous commencerons par présenter la démarche entreprise dans le cadre de deux projets de recherche complémentaires. Le première recherche a été réalisée dans le cadre de la thèse de doctorat d'Ellen Avard (2015) et a été soutenue par un ensemble de partenaires (l'Université Laval, le Conseil québécois de l'horticulture, le Village nordique de Kuujjuaq, l'Administration régionale Kativik et une équipe de bénévoles de Kuujjuaq). La deuxième recherche est celle de la thèse de doctorat d'Annie Lamalice, commencée en 2015 dans le cadre des activités de l'Observatoire hommes-milieux international (OHMI) du Nunavik ${ }^{1}$, dans la continuité du travail précédemment accompli. Dans les deux cas, une approche participative a été privilégiée, afin de créer un dialogue chercheurscitoyens autour des grands enjeux de la santé. Les différentes étapes franchies depuis 2009 seront présentées dans une analyse articulée autour des concepts de sécurité alimentaire culturelle et de souveraineté, basée sur les projets de serres communautaires de Kuujjuaq et d'Iqaluit et une étude de faisabilité à Kangiqsujuaq. Nous conclurons par une discussion de ce nouveau développement agricole nordique sous l'angle des retombées positives et des défis techniques et organisationnels auquel il doit faire face.

\section{La dimension culturelle de la sécurité et de la souveraineté alimentaires}

La sécurité alimentaire est une condition fondamentale pour une bonne santé (Wakelfield et al. 2015), que ce soit dans sa composante physique aussi bien qu'émotionnelle et mentale. Les problèmes générés par l'insécurité alimentaire comprennent des impacts sur la santé tels qu'anémie et carences alimentaires, mais ils peuvent aussi susciter exclusion sociale, sentiments de culpabilité et de honte, détresse, diminution de la capacité d'apprentissage et dépression (Ford 2009; Holzman 2011; Lambden et al. 2006; Tarasuk 2001). Ces aspects n'ont pas empêché le concept de sécurité alimentaire d'être instrumentalisé par des intérêts commerciaux au point d'en exclure les notions d'accessibilité et de préférences alimentaires, du moins jusqu'à tout récemment (Madeley 2002; Wakefield et al. 2015).

Lors de la Conférence mondiale de l'Alimentation à Rome en 1974, la sécurité alimentaire a été définie comme "la disponibilité en tout temps d'un approvisionnement adéquat en produits alimentaires de base pour soutenir une expansion régulière de la consommation alimentaire et contrebalancer les fluctuations de la production et des prix»(ONU 1975). L'accent était alors mis sur l'intensification de la production mondiale, selon l'idée véhiculée à l'époque que l'augmentation de l'offre permettrait d'assurer la sécurité alimentaire d'une 
population croissante (Madeley 2002). En 1983, l'Organisation des Nations unies pour l'alimentation et l'agriculture (FAO) modifie la définition initiale pour y ajouter la notion d'accès économique et physique à la nourriture pour toutes les personnes à l'intérieur d'une communauté (FAO 2006). Ce nouveau concept met l'accent sur les enjeux de distribution, l'augmentation de la production n'étant effectivement pas suffisante si les aliments n'atteignent pas les personnes souffrant de la faim. Plus tard, en 2002, la FAO y intègre une nouvelle préoccupation concernant les barrières sociales à l'accès à la nourriture et met l'emphase sur la relation entre nourriture et vie active (faisant référence au nouveau problème de suralimentation et d'obésité) (ibid.). Plus récemment, d'autres dimensions ont été ajoutées à la définition initiale, telles que le fait que la nourriture doit être culturellement acceptable et que le système alimentaire est tenu d'être durable, tout en favorisant la souveraineté et la justice sociale (Hamm et Bellows 2003).

Au Nunavik, en 2004, lors de l'enquête de santé Qanuippitaa, 24\% des Inuit ont déclaré avoir manqué de nourriture au cours du mois précédent (Blanchet et Rochette 2008). Cette situation d'insécurité alimentaire grave est plus fréquente chez les Inuit n'ayant pas de travail ou dont le revenu est très faible. De plus, la prévalence de l'insécurité alimentaire est exacerbée à mesure que le nombre de personnes augmente dans la maisonnée. Plus récemment, Ruiz-Castell et al. (2015) ont rapporté que $62 \%$ des familles inuit de l'Arctique canadien vivaient dans des logements surpeuplés, ce qui les mettait en situation de risque d'insécurité alimentaire. De plus, $27 \%$ des familles avaient déclaré réduire la taille des portions aux repas de leurs enfants à cause du manque d'argent, et cette attitude était plus fréquente dans les maisonnées surpeuplées.

Ce survol rapide de l'évolution de la définition de la sécurité alimentaire reflète les échecs successifs des tentatives de résolution des problèmes nutritionnels à l'échelle mondiale et la prise en compte de plus en plus inévitable de l'acceptabilité culturelle et de la nécessaire durabilité des systèmes alimentaires. En outre, concernant plus spécifiquement les populations autochtones, Power (2008) propose que la notion actuelle de sécurité alimentaire soit élargie pour y inclure une nouvelle dimension, à savoir la "sécurité alimentaire culturelle». Selon cette perspective, la récolte de denrées alimentaires provenant des systèmes traditionnels est non seulement une clé de l'identité culturelle, de la santé et de la survie, mais également le principal moyen de transmission des valeurs culturelles, des compétences et de la spiritualité. Ainsi, les indicateurs de la sécurité alimentaire culturelle comprennent des niveaux de connaissance sur la nourriture traditionnelle, l'accès aux systèmes alimentaires traditionnels et la sécurité de ces aliments (ibid.).

Les cultures autochtones posent un regard holistique sur la santé, prenant en compte l'ensemble des composantes d'un individu et de son environnement pour déterminer la nature des problèmes et des solutions. Cette vision repose sur l'équilibre et la mise en relation des composantes mentales, sociales, physiques et spirituelles d'une personne (NAHO 2008). Le concept de la santé holistique s'étend au-delà de la sphère individuelle en associant l'individu à la famille, à la 
communauté et à la culture. Elle se fonde sur le respect, l'humilité, le partage, l'hospitalité. Toutes ces dimensions se reflètent dans le régime alimentaire culturel traditionnel. Il est donc essentiel de reconnaître que les facteurs déterminants de la santé et de la sécurité alimentaire des communautés inuit prennent racine dans leurs conditions de vie, façonnées par des forces culturelles, sociales, économiques et historiques (Reading et Wien 2009).

Le concept de souveraineté alimentaire est complémentaire de celui de sécurité alimentaire. Cette souveraineté se définit comme «le droit et le pouvoir d'un pays ou d'une communauté de déterminer la production, la distribution et la consommation de sa nourriture en fonction de ses goûts et de ses traditions" (Madeley 2002: 53). Au cour de cette notion se retrouvent le renforcement de la communauté et des moyens de subsistance, et la durabilité sociale et environnementale des activités de production, de consommation et de distribution d'aliments nutritifs et culturellement acceptables (Desmarais et Wittman 2014). La souveraineté alimentaire est plus holistique que la sécurité alimentaire, car elle questionne le type de nourriture, ainsi que l'endroit, la façon et l'échelle à laquelle elle est produite, distribuée et consommée (Desmarais et Wittman 2014). Ainsi, en contexte autochtone, elle tient compte du caractère sacré de la nourriture et du réseau de relations qui la relie au monde naturel (FSC 2011).

Aujourd'hui, alors que le transport vers le Nord est plus rapide et efficace que jamais, les communautés inuit réalisent que le système alimentaire conventionnel et mondialisé a des défauts inhérents (Avard 2015), défauts de plus en plus apparents lorsqu'on s'éloigne des centres de production et de distribution. Non seulement le transport génère un coût environnemental énorme pour l'importation d'aliments venant d'ailleurs et des problèmes de conservation des aliments frais, mais également un risque de pénurie alimentaire aigüe imminent, en cas de rupture dans la chaîne de transport qui relie les villages nordiques à leurs bases d'approvisionnement du Sud (ibid.). Inversement, les expériences de production locale de nourriture révèlent une meilleure réponse aux besoins de la communauté, car elles priorisent les acteurs locaux et leur environnement, tout en renforçant l'identité sociale et la cohésion (Anderson et Cook 1999). Les projets de serres nordiques pourraient donc contribuer à renforcer la souveraineté alimentaire au Nunavik et au Nunavut tout en répondant aux besoins de la sécurité alimentaire culturelle.

La construction d'une nouvelle stratégie alimentaire durable dans l'Arctique canadien doit donc s'intéresser à la sécurité et à la souveraineté alimentaires, en tenant compte des perceptions et des besoins spécifiques de la population inuit et de la dimension culturelle de la sécurité alimentaire. Certaines politiques gouvernementales soutiennent le développement d'une production bioalimentaire dans le Nord du Canada. C'est le cas au Nunavik, dans le cadre du Plan Nord qui comporte un volet bioalimentaire, dont l'une des priorités pour le plan d'action 2015-2020 est de "fournir un appui adapté aux collectivités locales et autochtones isolées qui investissent en vue d'augmenter la production locale d'aliments pour réduire leur dépendance et le coût élevé de l'alimentation" 
(Société du Plan Nord 2014). Il s'agit, par exemple, d'appuyer les initiatives d'implantation de cultures sous abris (serres, tunnels, etc.). Il existe plusieurs expériences agricoles sous serre à travers le Nord canadien. Elles prennent la forme de jardins communautaires à Kuujjuaq, Iqaluit, Naujaat, Inuvik et Dawson City, d'opérations commerciales à Inuvik et Carmacks, ou encore de serres expérimentales dédiées à la recherche à Kuujjuarapik et à Devon Island. Le projet à l'étude à Kangiqsujuaq s'inscrit ainsi dans une mouvance nordcanadienne soutenue par la politique gouvernementale québécoise.

\section{Une recherche-action participative}

L'approche utilisée dans ces deux projets est une forme de recherche-action appelée "recherche participative basée dans la communauté»². Cette approche implique à égalité les chercheurs et les partenaires locaux, et ce, à toutes les étapes du processus de recherche, de la conception du projet jusqu'à la diffusion des résultats, en passant par l'analyse et l'interprétation des données. C'est une forme de recherche souple, qui s'adapte au contexte local (Avard 2015; Chevalier et al. 2013). Le plus souvent appliquée dans les domaines de la santé publique et du travail social, cette méthodologie est apparue au cours des dernières décennies comme un paradigme alternatif de recherche qui combine l'éducation et l'action sociale (Bourassa et al. 2007; Wallerstein et Duran 2006). Cette façon innovante et flexible de conduire la recherche s'est construite sur les fondations posées par Paulo Freire et Kurt Lewin (Flicker et al. 2008; Israel et al. 2001; Wallerstein et Duran 2003). Elle se concentre sur les relations entre partenaires universitaires et communautaires, en mettant l'accent sur les principes de co-apprentissage, de bénéfice mutuel et d'engagement à long terme (Avard 2015).

Les travaux sur le terrain de la première recherche, celle de la thèse de doctorat d'Ellen Avard (à l'Université Laval), se sont échelonnés de 2009 à 2013 autour du projet de serre communautaire de Kuujjuaq. Réalisée sous forme d'étude de cas, cette recherche-action a évalué l'acceptabilité sociale de tels projets au Nunavik, puis a contribué à leur développement ainsi qu'à une meilleure compréhension de leur contribution à la construction d'un système alimentaire durable. La collecte de données a combiné différentes méthodologies. À l'automne 2009, 31 entrevues dirigées et 36 entrevues semi-dirigées ont été réalisées auprès de 67 personnes à Kuujjuaq dans le cadre des consultations sur l'acceptabilité sociale des projets de serres. Quatre ans plus tard, au printemps 2013, 32 entrevues dirigées et 11 entrevues semi-dirigées ont été réalisées avec les acteurs du développement serricole à Kuujjuaq. De plus, les longs séjours réalisés par la chercheure doctorale et son rôle de coordonnatrice du projet de serre durant ses deux premiers étés, en 2010 et 2011, lui ont permis d'accéder à une compréhension approfondie de la thématique, par exemple, à travers de

2. En anglais: community-based participatory research (CBPR). 
nombreuses conversations informelles. Les données obtenues lors des entrevues lui ont permis de générer de nouvelles connaissances, tout en répondant concrètement aux besoins exprimés par les Kuujjuamiut.

Le succès de Kuujjuaq a encouragé d'autres initiatives horticoles, comme celle de Kangiqsujuaq, toujours au Nunavik. C'est à partir du souhait explicite de cette communauté qu'un nouveau projet de recherche a été mis en place en 2015, dans le cadre des activités de l'OHMI du Nunavik et de la thèse de doctorat d'Annie Lamalice (entreprise conjointement à l'Université de Montpellier et à l'Université de Montréal). S'appuyant sur les résultats de la thèse d'Avard (2015), cette recherche s'est, jusqu'à aujourd'hui, penchée sur les projets de serres communautaires d'Iqaluit (Nunavut) et de Kuujjuaq, afin d'en comprendre les spécificités techniques et organisationnelles, dans le but de réaliser une étude de faisabilité pour le développement d'un nouveau projet à Kangiqsujuaq. Cette première phase exploratoire a été réalisée lors d'un séjour de recherche à Iqaluit, Kuujjuaq et Kangiqsujuaq en 2015. La prochaine étape de ce projet est l'organisation d'ateliers participatifs à Kuujjuaq et à Kangiqsujuaq dans le but d'approfondir les connaissances sur le potentiel des projets d'agriculture nordique pour le développement d'un système alimentaire durable, tout en contribuant à l'autonomisation des participants locaux.

Comme le soulignent Rastoin et Ghersi (2010), le système alimentaire est un objet d'études qui touche à la vie et à la société humaine dans sa globalité, faisant de l'interdisciplinarité un impératif pour traiter de la thématique de l'alimentation. C'est ce que fait ce projet, en associant géographie de la santé, ethnobotanique, nutrition, santé et ingénierie thermique, pour répondre à la complexité de la sécurité alimentaire dans les villages du Nunavik, en développant une agriculture nordique.

\section{Les différentes étapes}

En 2009 , la première étape du projet d'Avard a consisté à étudier l'acceptabilité sociale de projets de serres au Nunavik pour répondre à la problématique de la sécurité alimentaire mais aussi à quelques défis de nature socioéconomique (comme par exemple l'important taux de décrochage scolaire et la forte incidence du chômage). Ce travail, basé sur des entretiens et questionnaires, s'est soldé par un intérêt marqué du village de Kuujjuaq pour accueillir un projet-pilote sous la forme de petits projets horticoles en serre. Une première serre (Figures 1 et 2), construite dans les années 1990 dans le cadre d'un projet de verdissement de la ville, restait utilisée par quelques jardiniers autonomes. Des consultations publiques organisées à Kuujjuaq en 2010 ont permis de présenter les résultats de l'étude préliminaire, de confirmer l'intérêt de la population et de sécuriser le financement pour le lancement du projet grâce à l'appui du village nordique de Kuujjuaq, du Conseil québécois de l'horticulture, du ministère de l'Agriculture, des Pêcheries et de l'Alimentation du Québec (MAPAQ) et de l'Administration régionale Kativik (Avard 2015). 

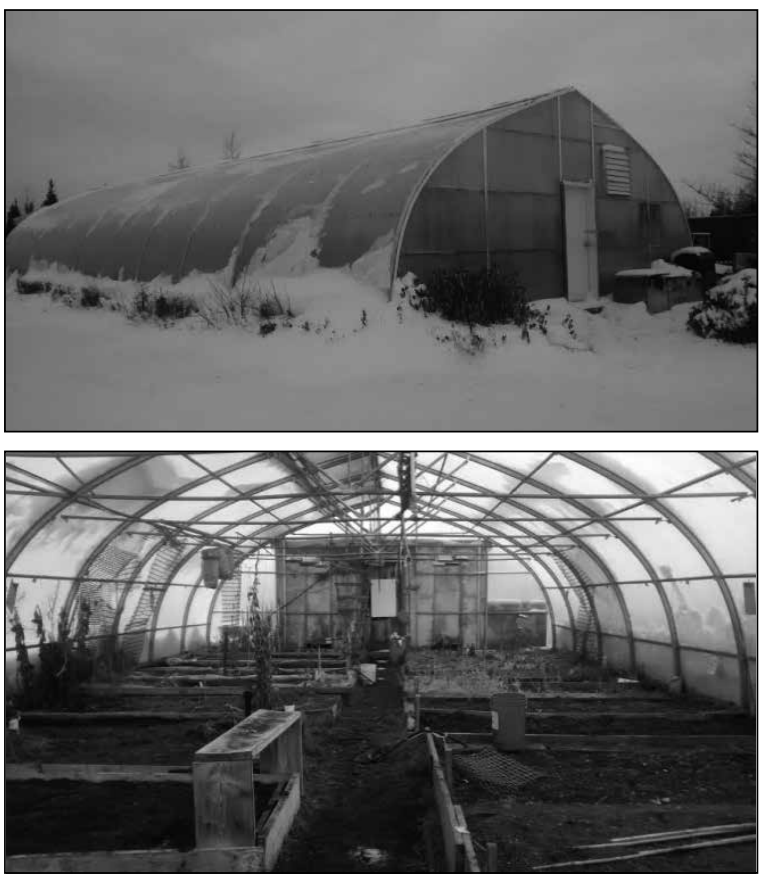

FIGURES 1 et 2. Extérieur et intérieur de la première serre de Kuujjuaq (construite dans les années 1990), en octobre 2015. Photos: Annie Lamalice.

C'est en 2011 qu'est lancée la phase I du projet de serre de Kuujjuaq. La chercheure doctorale est alors coordonnatrice de huit microprojets d'horticulture sous serre, tout au long de la saison de culture. En collaboration avec des volontaires locaux, le village de Kuujjuaq et Hébergement communautaire Ungava $^{3}$, des initiatives sont ainsi développées: un jardin communautaire, un projet de compost, un projet "d'hortithérapie», un jardin expérimental de pommes de terre, la planification de la construction de la deuxième serre, le développement d'un programme scolaire avec la Commission scolaire Kativik et des essais hydroponiques. En parallèle, une collecte de données est organisée auprès des jardiniers de la serre pour déterminer quels légumes sont les mieux adaptés à un projet horticole en serre dans le contexte climatique de Kuujjuaq. En 2012, la phase II du projet débute. Les huit microprojets se regroupent sous l'égide du "Projet de serre de Kuujjuaq" qui connaît des avancées importantes, notamment en ce qui concerne le projet de compostage auquel se sont associés les épiceries et restaurants de Kuujjuaq, l'Administration régionale Kativik et Hébergement communautaire Ungava. Face au succès global de la démarche, la construction d'une seconde serre est engagée en 2012 (Figures 3 et 4). Les deux

3. Il s'agit d'une résidence pour personnes souffrant de problèmes de santé mentale ou de déficience intellectuelle. 

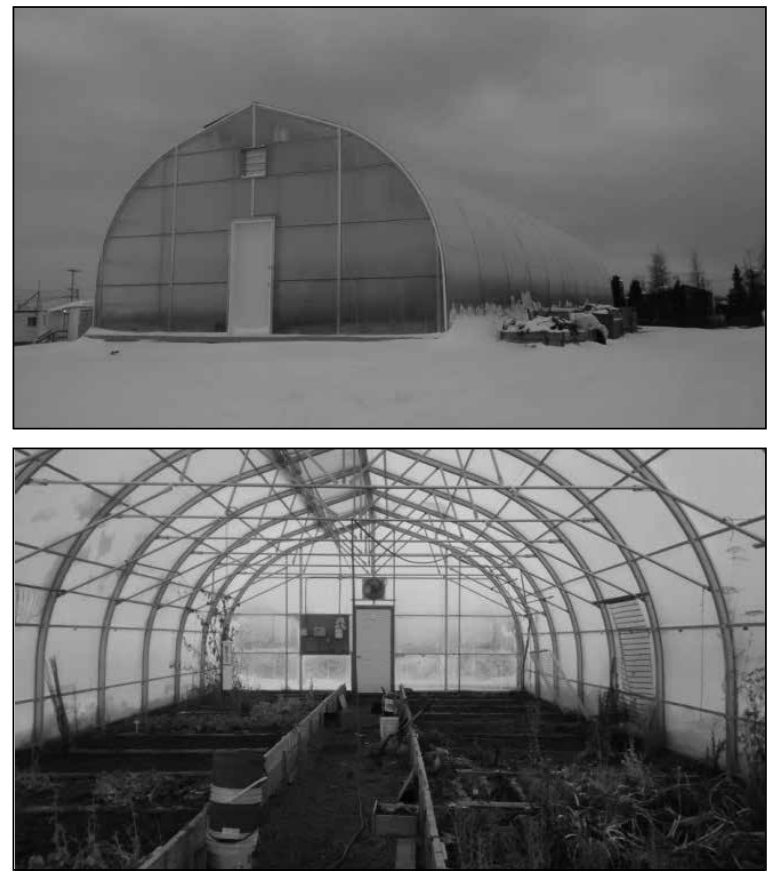

FIGURES 3 et 4. Extérieur et intérieur de la seconde serre de Kuujjuaq (construite à partir de 2012) en octobre 2015. Photos: Annie Lamalice.

bâtiments, construits côte à côte légèrement en marge de la ville, ont chacun une superficie de $133 \mathrm{~m}^{2}$. Les serres sont divisées en 46 lots individuels d'environ $4 \mathrm{~m}^{2}$ attribués par tirage au sort aux individus ou familles intéressés à jardiner pour la saison de culture annuelle qui va généralement de mi-mai à septembre. Deux petites serres ont aussi été construites sur le terrain des garderies d'enfants afin de permettre aux petits de se familiariser avec le jardinage. C'est en 2013 que se termine la collecte de données à Kuujjuaq avec une série d'entretiens dont l'objectif est d'évaluer les retombées du projet.

En continuité avec le travail accompli entre 2009 et 2013, un nouveau programme de recherche démarre en 2015 à Kangiqsujuaq. Ce petit village, situé à $10 \mathrm{~km}$ du détroit d'Hudson sur la rive sud-est de la baie de Wakeham, avait en effet manifesté le souhait de développer son propre projet de serre. Le démarrage du nouveau projet de recherche est officialisé par un état des lieux conduit durant les mois d'octobre et novembre 2015, s'appuyant sur une analyse des modes d'organisation et de fonctionnement de deux projets de serres communautaires existants à Iqaluit et à Kuujjuaq, puis sur une étude de faisabilité à Kangiqsujuaq. Ces communautés ont été retenues principalement pour la similitude des conditions bioclimatiques qu'elles présentent par rapport à Kangiqsujuaq, où le nouveau projet de serre est envisagé. Les dynamiques 

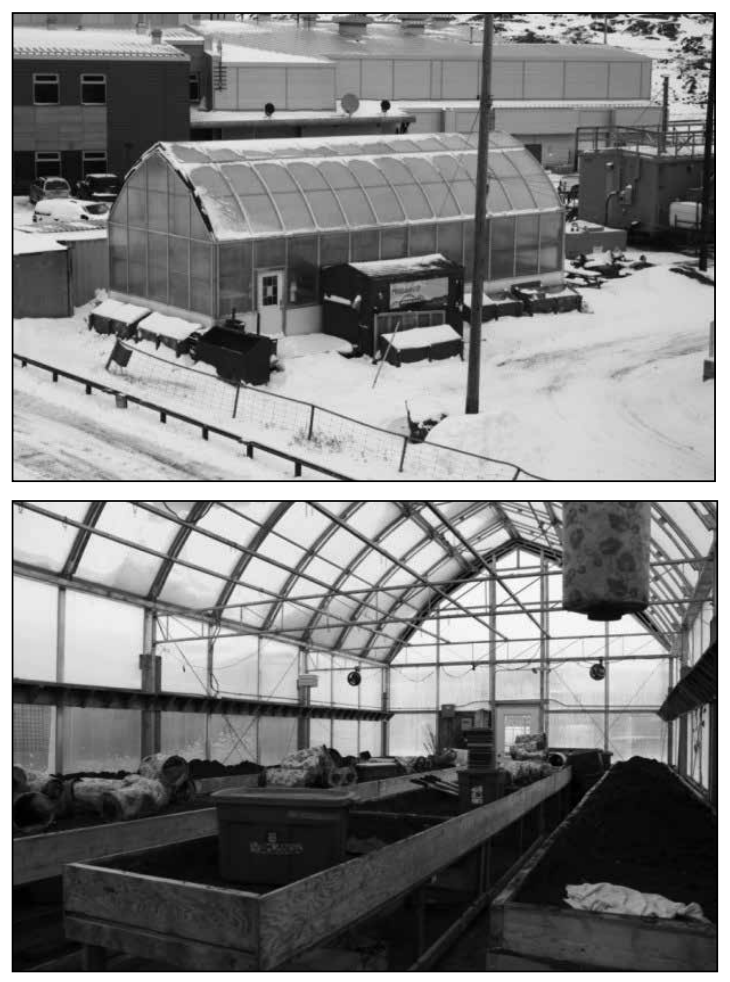

FIGURES 5 et 6 . Extérieur et intérieur de la serre d'Iqaluit (construite en 2007) en octobre 2015. Photos: Annie Lamalice.

sociales y sont toutefois assez différentes, Iqaluit et Kuujjuaq comportant des populations plus nombreuses et aux origines ethniques plus diversifiées qu'à Kangiqsujuaq où $93 \%$ des 690 habitants ont déclaré être Inuit lors du dernier recensement (Statistique Canada 2014).

Contrairement à Kangiqsujuaq, les projets de serres à Kuujjuaq et à Iqaluit ont été initiés par des travailleurs provenant du Sud du Canada. Lors de ce terrain, des consultations ont été réalisées avec les bénévoles impliqués dans la gestion des projets de serres d'Iqaluit et de Kuujjuaq et des acteurs intéressés par la thématique, à la Régie régionale de la Santé et des Services sociaux, puis au sein de l'Administration régionale Kativik et du centre de recherche du Nunavik. Depuis 2007, Iqaluit est dotée d'une serre communautaire d'une superficie de $90 \mathrm{~m}^{2}$ (Figures 5 et 6 ). Elle compte entre 70 et 80 membres qui se répartissent l'espace intérieur en cultivant dans de longs bacs de culture hors-sol durant une saison d'environ 12 semaines. Une entrevue et une visite de la serre ont été réalisées avec la chargée des communications du conseil d'administration. À Kangiqsujuaq, des rencontres avec les personnes intéressées par le développement d'un projet de serre communautaire ont été organisées afin de 
confirmer l'intérêt de ce village et d'étudier les différentes possibilités. Des consultations ont ainsi pu être réalisés avec le maire, le gestionnaire du village, le propriétaire d'une serre privée ainsi que des travailleuses des secteurs de la santé et de l'éducation.

\section{Résultats}

Les deux projets de recherche mènent au même constat: la perspective d'installer des serres nordiques et de développer de nouvelles stratégies agricoles durables dans les communautés inuit est jugée très positive (Avard 2015). Les serres permettent de renforcer la sécurité alimentaire en contribuant à améliorer l'apport nutritionnel. Il est évident que ces données demandent à être consolidées sur plusieurs années et qu'il est nécessaire d'évaluer de manière approfondie les taux de consommation des récoltes, mais les premières estimations sont très encourageantes. Les retombées diverses sont décrites ci-après, ainsi que les obstacles rencontrés dans la pleine réalisation des objectifs des projets de serres de Kuujjuaq et d'Iqaluit, et les défis techniques et organisationnels à prendre en compte pour la mise en place d'un nouveau projet ailleurs au Nunavik.

\section{Productions végétales de la serre et amélioration des apports nutritionnels}

De nombreuses espèces végétales ont été testées dans les serres communautaires de Kuujjuaq et d'Iqaluit. Les meilleurs résultats ont été obtenus avec les légumes verts en feuilles (laitues, bettes à carde, bok choy), ainsi que les herbes aromatiques et les radis. Ces végétaux y poussent exceptionnellement bien et ont l'avantage de permettre plusieurs récoltes, même durant la petite saison de croissance des climats arctiques et subarctiques. Ensuite, les pommes de terre, oignons, carottes et betteraves sont faciles à cultiver et produisent des légumes de taille respectable (Avard 2015). En revanche, les plantes qui aiment la chaleur, telles que les tomates, se révèlent plus difficiles à faire pousser si elles n'ont pas été semées chez des particuliers bien avant le début de la saison. En extrapolant les données récoltées par Avard entre 2010 et 2011, chacune des serres de Kuujjuaq (d'une superficie de 7,6 x 18,3 mètres ou 25 x 60 pieds) a le potentiel de produire au moins $300 \mathrm{~kg}$ de nourriture par an, ce qui constitue un apport nutritionnel intéressant pour les jardiniers et leurs familles, sachant que la consommation moyenne de légumes (sans compter les pommes de terre) est de $0,78 \mathrm{~kg}$ par adulte et par semaine au Canada (Duchemin et al. 2010).

À titre d'exemple, à Iqaluit, en 2014, 60\% des légumes cultivés étaient des laitues et légumes en feuille; en plus petite quantité, on y trouvait aussi du chou kale, des haricots, des pois, des radis et des carottes. La richesse de ces aliments en minéraux et vitamines (Souci et al. 2008) permet de couvrir en grande partie, voire en totalité, les quantités recommandées. En outre, ces légumes sont vecteurs de micronutriments tels que les phytostérols (Wu et al. 2009) ou les glycosinolates (Dinkova-Kostova et Kostov 2012; Howes et Simmonds 2014; 
Kapusta-Duch et al. 2012), pour lesquels il n'existe pas actuellement de recommandations, mais dont les vertus sont avérées. En plus de leurs qualités nutritionnelles, les fruits et légumes issus de la serre locale permettent une préservation des vitamines qui sont détruites en cas de conservation longue des aliments (ce qui est fréquemment le cas des produits de supermarché), en plus d'offrir une fraîcheur inégalable et des coûts financiers (pour la famille) et environnementaux moins élevés (Avard 2015). Dans le cas du projet de serre de Kuujjuaq, les 46 lots sont cultivés de façon individuelle et une grande variété de plantes y pousse chaque été en fonction des préférences des jardiniers. Le recensement des cultures et leur contribution à l'apport nutritionnel des participants restent à être évalués.

\section{Retombées socioculturelles des projets de serres}

Les entrevues réalisées en 2013 par Avard (2015) révèlent que les principaux avantages perçus par les participants sont les bénéfices à long terme pour la santé physique et psychologique, ce dont témoignent des propos tels que: "c'est bon pour l'esprit et pour le corps ${ }^{4}$ (entrevue 27); ou bien "[le jardinage] fait sortir les gens de leur maison, ça les pousse à interagir avec les autres [...] ça fait du bien au moral" (entrevue 25). Autre effet positif, les participants éprouvaient un sentiment de fierté et d'accomplissement qu'ils ont mentionné dans la majorité des entretiens. Ce sentiment était associé au fait de faire pousser soi-même les aliments consommés par sa famille, mais aussi au fait que le projet de serre permet "une autonomie en termes de production alimentaire menant à une plus grande sécurité alimentaire» (entrevue 5) ainsi qu'«une dépendance moindre envers les denrées du Sud" (entrevues 14 et 32). À Kuujjuaq, l'école et différents groupes de la communauté ont leur parcelle de jardinage attitrée dans la serre. Les opportunités créées pour les enfants et les jeunes par les projets de jardinage sont perçues comme un bénéfice, puisque cela "permet aux enfants d'apprendre et de voir d'où vient la nourriture, dès la première étape» (entrevue 29). Finalement, des retombées sociales plus larges des projets de serres ont aussi été mentionnées, telles que le renforcement du tissu social (entrevues 13 et 14), la création d'emploi et la réinsertion sociale (entrevues 17 et 26) ainsi que la transmission des connaissances (entrevue 19).

\section{Acceptabilité sociale d'un projet de serre}

Les entrevues de 2013 ont aussi montré que les aliments issus de la serre, bien que l'agriculture ne soit pas une activité traditionnelle, peuvent être acceptables culturellement. Plusieurs ont fait le rapprochement entre l'agriculture et l'activité traditionnelle qu'est la cueillette de petits fruits: "cueillir des petits fruits est très similaire [...] une sorte d'agriculture passive» (entrevue 6); "les

4. Toutes les citations d'entrevues sont des traductions libres de l'anglais. 
projets agricoles contribueraient seulement à amener la cueillette à autre niveau " (entrevue 23); "l'agriculture est une extension logique de la cueillette de petits fruits et donc traditionnelle jusqu'à un certain point" (entrevue 13). Le fait que l'agriculture ne soit pas une activité traditionnelle ne signifie pas que les Inuit ne veulent pas l'essayer (entrevue 29): "vivre dans des villes et conduire des véhicules [ne sont pas des activités traditionnelles]... les peuples à travers le monde font de l'agriculture, pourquoi pas les Inuit?» (entrevue 6). Finalement, le thème de l'autosuffisance alimentaire est apparu important pour les participants, qui voient dans les projets de serres une occasion de retrouver une autonomie perdue au cours des dernières décennies (entrevue 19). Le jardinage est ainsi perçu par certains participants comme "une nouvelle approche pour une façon ancienne de nourrir les familles, une nouvelle méthode à laquelle nous pouvons nous identifier» (entrevue 1).

\section{Difficultés exprimées}

Les retombées positives du projet de serre de Kuujjuaq sont donc multiples et son potentiel pour améliorer la qualité de vie n'a pas échappé aux Kuujjuamiut. Cependant, les difficultés rencontrées par les promoteurs demeurent et de nombreux obstacles à l'élaboration d'initiatives alimentaires locales sont à déplorer. (Il faut néanmoins souligner que ces difficultés sont observées dans les jardins communautaires sous toutes les latitudes et ne sont pas spécifiques au contexte nordique.) Les principales difficultés sont le déficit de financement pour les projets à petite échelle et le manque de connaissances techniques. Le défaut de supervision des opérations au jour le jour pose également problème et est souvent dû à l'absence d'un travailleur rémunéré. L'entretien des lieux et la remise en activité annuelle, y compris en ce qui concerne des actions relativement simples, telles que la mise à jour des informations de contact pour tous les jardiniers, la commande d'eau, la centralisation et la communication des informations relatives à la serre, font parfois défaut (Avard 2015).

À Iqaluit se rencontrent ponctuellement différentes difficultés organisationnelles. Cela s'explique principalement par le roulement important des personnes impliquées. En effet, la plupart des participants sont des travailleurs temporaires venus du Sud qui s'engagent dans le projet pour quelques mois ou quelques années, avant de quitter la ville. Cela soulève la problématique de la faible inclusion de la communauté inuit dans le projet d'Iqaluit, tel qu'observé lors du séjour de recherche en octobre 2015 et ainsi que l'avait déjà constaté Holzman (2011) auparavant. À l'inverse, les projets de Kuujjuaq ont un rayonnement important dans la communauté, y compris auprès des Inuit, qui représentent environ la moitié des jardiniers.

\section{Pistes d'amélioration}

Les répondants ont partagé différentes idées et commentaires lors des entrevues finales réalisées à Kuujjuaq en 2013, en vue d'améliorer les projets de serres et de créer davantage de liens avec la communauté (Tableau 1). 
Premièrement, pour en pérenniser le succès, les initiatives devraient être conduites par la communauté. L'adhésion des personnes en serait facilitée et permettrait d'associer activement les enfants et les jeunes. Ensuite, plusieurs résidents seraient intéressés à faire l'acquisition d'une petite serre privée et aimeraient que des aides soient mises en place en ce sens. D'autre part, dans le cadre d'une démarche écologique, certains souhaitaient que la valorisation des déchets locaux soit maximisée, ce qui impliquerait la construction ou l'achat de bacs à compost que les résidents pourraient installer chez eux.

Par ailleurs, un partenariat avec la cuisine communautaire permettrait de créer des opportunités de découverte des nouveaux légumes et d'apprentissage pour les cuisiner. Finalement, de nombreux répondants ont manifesté un intérêt pour les plantes locales et leur potentiel pour améliorer l'aspect de la ville. L'idée serait de faire pousser des fleurs et plantes domestiques, notamment pour souligner certaines fêtes (la fête des mères, la Saint-Valentin et Pâques) (Avard 2015). Toutes ces idées émanant des participants reflètent l'enthousiasme et l'appropriation du projet par la population locale. Sur le plan technique, de nombreuses améliorations pourraient être apportées concernant l'approvisionnement en eau, l'isolation, la ventilation et le système de chauffage; le manque de connaissances sur ces paramètres ainsi que sur l'horticulture en général est la plus grande faiblesse du projet, selon les participants rencontrés en octobre 2015 par Lamalice.

\section{Avantages associés}

- Bénéfices à long-terme pour la santé physique et psychologique;

- Sentiment de fierté et d'accomplissement;

- Education et transmission du savoir;

- Gain en autonomie face aux importations;

- Renforcement du tissu social;

- Création d'emploi et réinsertion sociale.

Difficultés rencontrées

- Financement difficile à obtenir pour des projets à petite échelle;

- Failles dans la coordination et l'entretien en raison de l'absence d'un travailleur salarié:

- Adaptations techniques nécessaires face aux conditions bioclimatiques.

Pistes d'amélioration

- Mettre en place un système de transfert de connaissances;

- Inclure activement les enfants et les jeunes;

- Adaptations techniques: approvisionnement en eau, isolation, ventilation, système de chauffage;

- Valorisation des plantes locales;

- Valorisation des déchets locaux à plus grande échelle.

TABLEAU 1. Tableau récapitulatif des avantages, difficultés et pistes d'améliorations des projets de serres nordiques. 


\section{Quel futur pour les communautés?}

À Kangiqsujuaq, la population a manifesté un vif intérêt pour l'installation d'une serre communautaire. Ce souhait a été confirmé lors des consultations réalisées lors du séjour de recherche en novembre 2015. Deux scénarios sont envisagés par la municipalité: la transformation du bâtiment de l'actuelle piscine en serre (Figure 7), ou la construction d'un nouveau bâtiment. Dans les deux cas, il faudra tenir compte des difficultés techniques pour que la nouvelle serre soit adaptée au climat local. Dans la construction, les paramètres importants sont l'isolation du plancher et idéalement du mur nord, un système d'approvisionnement en eau qui permette d'éviter le gel durant toute la saison de culture et une gestion automatique de l'hygrométrie et de la température. En ce qui concerne le sol, la méthode "hors-sol" telle qu'utilisée à Iqaluit devrait être privilégiée pour éviter le contact avec le pergélisol. Il faut aussi réfléchir au meilleur système d'approvisionnement en terreau, à la gestion des ravageurs et à la mise sur pied d'un projet de compostage. Le choix des plantes doit être étudié attentivement et un équilibre doit être trouvé entre nécessité nutritionnelle, rendement, traditions et facilité de cultures. Finalement, il est urgent d'organiser un transfert de connaissances vers la population locale, afin de valoriser l'espace de jardinage, d'optimiser les productions et d'éviter que les participants se découragent. En ce qui concerne les défis organisationnels, la future équipe du projet de Kangiqsujuaq devra réfléchir à des solutions pour éviter les problèmes vécus à Iqaluit (tels que le roulement important des responsables de la serre) et ainsi assurer la pérennisation et le rayonnement de la serre dans la communauté.

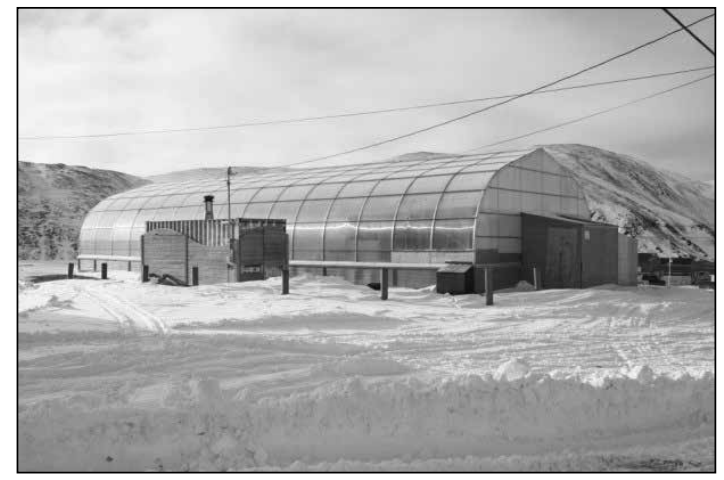

FIGURE 7. Actuel bâtiment de la piscine de Kangiqsujuaq en novembre 2015 qui pourrait être converti en serre. Photo: Annie Lamalice.

À Kuujjuaq, l'installation de serres a suscité de nouvelles initiatives tout à fait complémentaires. Un projet de compostage est géré par une association de jeunes. Anguviaq, l'Association des chasseurs, pêcheurs et piégeurs de Kuujjuaq, en partenariat avec l'Administration régionale Kativik, a construit un poulailler 
accueillant une centaine de poules pondeuses depuis 2015. Un élevage de lapins est projeté pour 2016. Ces initiatives "peuvent être bénéfiques pour les chasseurs. Cela signifie que si nous manquons de caribous ou de baleines, nous pourrons aider à diminuer le coût de la vie dans le Nord" (témoignage de Thomas Shea, président d'Anguviaq, in Taylor 2014). Ces projets représentent donc une protection en cas de pénurie alimentaire et un regain d'autonomie vis-à-vis des importations du Sud.

\section{Discussion}

L'insécurité alimentaire des Inuit relève de facteurs complexes et variés et ne pourra être résolue qu'à moins de mener différentes stratégies en parallèle. Le développement des projets horticoles qui contribueront au bien-être et à la santé des Inuit fait partie de ces stratégies. L'alimentation traditionnelle est fondamentale pour le maintien de la culture et des rapports de l'homme avec son milieu. La Régie régionale de la Santé et des Services sociaux du Nunavik encourage d'ailleurs la pratique des activités de subsistance et la consommation des aliments du terroir, comme en témoigne le guide alimentaire qu'elle a rédigé (Figure 8). Néanmoins, le coût élevé du matériel de chasse et de pêche, le travail salarié chronophage et les changements climatiques sont des facteurs qui compliquent l'accès aux activités de subsistance (Ford 2009; Furgal et Séguin 2006; Huet et al. 2012). Par ailleurs, la nourriture provenant des mammifères, oiseaux et poissons représente la principale source d'exposition aux contaminants environnementaux (AMAP 2009; Damman et al. 2008)

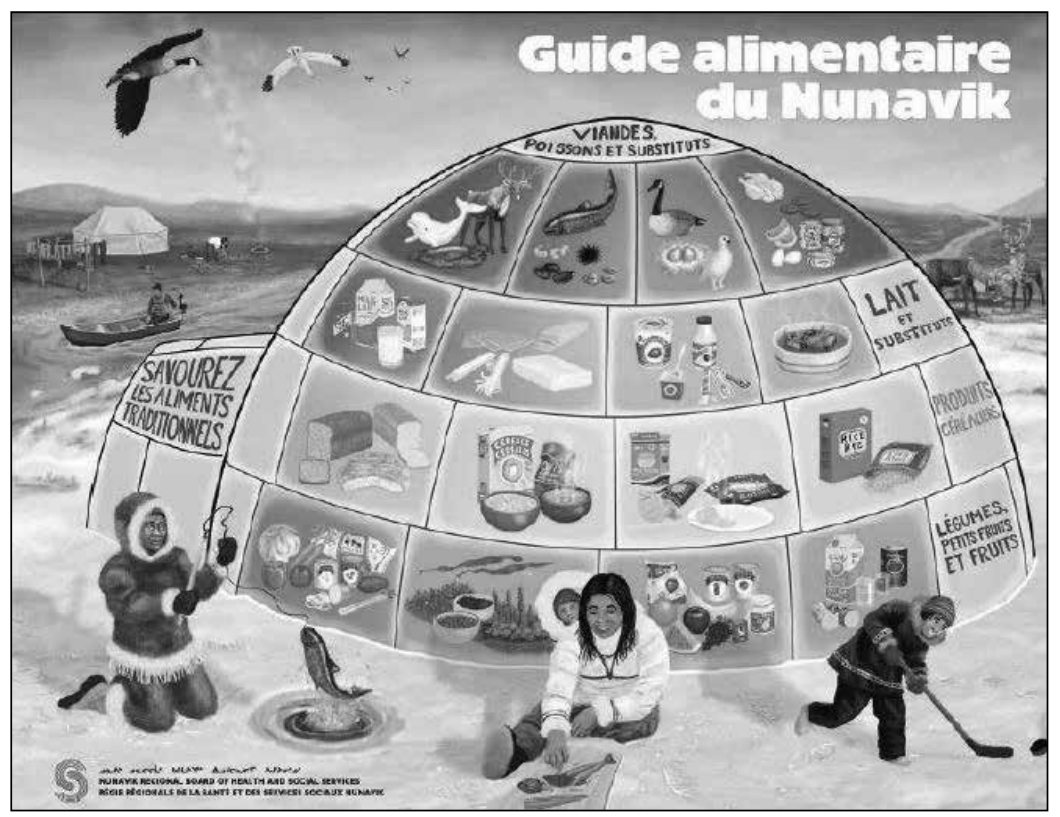

FIGURE 8. Le Guide alimentaire du Nunavik. Source: RRSSSN (n.d.). 
Les scientifiques s'accordent à penser que la sécurité alimentaire globale s'améliorerait avec un meilleur accès aux aliments du marché de qualité (Chan et al. 2006). Jusqu'ici, le jumelage de l'alimentation traditionnelle avec les aliments commerciaux n'a permis de répondre ni aux besoins nutritionnels ni aux impératifs culturels des Inuit (AMAP 2009). En somme, de nouvelles solutions doivent être proposées et nos données préliminaires nous laissent penser que le développement d'une production agroalimentaire à l'échelle locale a le potentiel de devenir l'une de ces solutions, notamment parce qu'une fourniture locale de nourriture permet de répondre beaucoup plus efficacement aux besoins de la communauté. En effet, elle permet de prioriser les acteurs locaux et leur milieu, tout en renforçant l'identité et la cohésion sociale (Anderson et Cook 1999). Cela correspond par ailleurs aux volontés exprimées par les Inuit dans le cadre du Plan Nunavik (2014). Comme le suggèrent Loring et Gerlach (2010), l'agriculture nordique ne doit pas être envisagée comme un moyen de subsistance exclusif, mais comme l'un des éléments d'une stratégie alimentaire diversifiée et structurante socialement, conclusion à laquelle parvient également Avard (2015).

Le développement d'une production locale peut favoriser la réconciliation des deux systèmes alimentaires (traditionnel et commercial) en permettant aux Inuit de s'approprier de nouvelles méthodes d'approvisionnement, tout en regagnant une autonomie et une souveraineté sur le plan alimentaire. Le raccourcissement de la chaîne alimentaire permet de combattre l'anonymat du système agro-industriel et implique souvent un contact plus direct entre le producteur et le consommateur (Renting et al. 2003). Les projets de serres permettent de regagner une plus grande autonomie sur le plan de l'alimentation, autonomie qui avait fortement diminué avec l'installation des épiceries dans tous les villages du Nunavik.

Les projets de serres peuvent aussi valoriser les savoirs écologiques traditionnels en y incluant la culture de plantes du terroir. Cette idée, suggérée par les Inuit de Kuujjuaq (Avard 2015) et de Kangiqsujuaq (consultations réalisées en novembre 2015), présente de nombreux avantages. Tout d'abord, cela permettrait de favoriser la transmission des savoirs liés aux plantes et de promouvoir les liens intergénérationnels. En outre, la culture de ces plantes permettrait aux personnes qui, pour diverses raisons, n'ont pas l'occasion de prendre part à des activités de cueillette à l'extérieur du village, d'en profiter également. Une telle pratique pourrait aussi servir un objectif pédagogique d'éducation des enfants des garderies et des écoles. Par ailleurs, l'apport pour la sécurité alimentaire n'est pas négligeable. En effet, des études (Kunhlein 2014; Powell et al. 2013) ont montré que la consommation de plantes locales, même si elles ne contribuent pas de façon significative aux besoins énergétiques quotidiens, peuvent tout de même apporter des quantités importantes de vitamines et de minéraux. Finalement, la culture d'espèces adaptées au climat 
local garantirait une meilleure réussite que celle de plantes exogènes introduites artificiellement et non adaptées au climat.

En conclusion, les projets de serres en cours de développement peuvent être socialement et culturellement acceptables pour la population du Nunavik, comme le démontrent les études-pilotes de Kuujjuaq et l'accueil positif d'un nouveau projet à Kangiqsujuaq. Les projets de serres permettent une approche alternative pour la sécurité alimentaire au Nunavik et peuvent concrètement contribuer au développement durable des collectivités de l'Arctique. Ils contribuent non seulement à l'amélioration de la qualité de vie et de la santé en apportant des produits sains à l'alimentation, mais aussi au bien-être des populations en favorisant l'autonomisation et un sentiment de fierté des acteurs locaux, tout en ouvrant diverses possibilités pour des projets d'éducation, de réinsertion, d'hortithérapie et d'embellissement de la ville.

\section{Remerciements}

Nous remercions les villages nordiques de Kuujjuaq et de Kangiqsujuaq d'avoir accepté que s'y déroulent nos recherches, ainsi que les organismes suivants qui ont contribué à leur financement: le Conseil québécois de l'horticulture, le Centre de recherche du Nunavik (Makivik Corporation), le Fond de recherche du Québec-Société et culture (FRQSC), le Conseil de recherches en sciences humaines du Canada (CRSH), l'Administration régionale Kativik, l'Association universitaire canadienne d'études nordiques (AUCEN), le Laboratoire d'Excellence "Dispositif de recherche interdisciplinaire sur les interactions hommes-milieux" (Labex DRIIHM) et l'Observatoire hommes-milieux international (OHMI) du Nunavik, ainsi que le Ministère de l'Agriculture, des Pêcheries et de l'Alimentation du Québec.

\section{Références}

ABEDI, Elahe et Mohammad Ali SAHARI

2014 Long-chain polyunsaturated fatty acid sources and evaluation of their nutritional and functional properties. Food Science and Nutrition, 2(5): 443-463.

AMAP (ARCTIC MONITORING AND ASSESSMENT PROGRAMME

2009 AMAP assessment 2009: Human Health in the Arctic, Oslo, Arctic Monitoring and Assessment Programme.

ANDERSON, Molly D. et John T. COOK

1999 Community food security: practice in need of theory?, Agriculture and Human Values, 16(2): 141-150.

AVARD, Ellen

2015 Northern Greenhouses: An Alternative Local Food Provisioning Strategy for Nunavik, thèse de doctorat, Université Laval, Québec. 
BLANCHET, Carole, Éric DEWAILLY, Pierre AYOTTE, Suzanne BRUNEAU, Olivier RECEVEUR et Bruce John HOLUB

2000 Contribution of selected traditional and market foods to the diet of Nunavik Inuit women, Revue canadienne de la pratique et de la recherche en diététique, 61(2): 50-59.

BLANCHET, Carole et Louis ROCHETTE

2008 Nutrition and food consumption among the Inuit of Nunavik, Nunavik Inuit Health Survey 2004, Qanuippitaa? How are we?, Québec, Institut national de santé publique du Québec et Régie régionale de la santé et des services sociaux, Nunavik (en ligne, https ://www.inspq.qc.ca/pdf/publications/762_ESI_Nutrition_Report_MA.pdf).

BOURASSA, Michelle, Louise BÉLAIR et Jacques CHEVALIER

2007 Les outils de la recherche participative, Éducation et Francophonie, 35(2): 1-11.

CHAN, Hing Man, Karen FEDIUK, Sue HAMILTON, Laura ROSTAS, Amy CAUGHEY,

Harriet KUHNLEIN, Grace M. EGELAND et Eric LORING

2006 Food security in Nunavut, Canada: barriers and recommendations, International Journal of Circumpolar Health, 2006, 65(5): 35-40.

CHÂTEAU-DEGAT, Marie-Ludivine, Éric DEWAILLY, Guylaine CHARBONNEAU, Elhadhi A. LAOUAN-SID, Angelo TREMBLAY et Grace M. EGELAND

2011 Obesity risks: towards an emerging Inuit pattern, International journal of circumpolar bealth, 70(2): 166-177.

CHEVALIER, Jacques M., Daniel. J. BUCKLES et Michelle BOURASSA

2013 Guide de la recherche-action, la planification et l'évaluation participatives, Ottawa, SAS2 Dialogue.

COUNIL, Émilie, Pierre JULIEN, Benoît LAMARCHE, Marie-Ludivine CHÂTEAU-DEGAT, Annie FERLAND et Eric DEWAILLY

2009 Association between trans-fatty acids in erythrocytes and pro-atherogenic lipid profiles among Canadian Inuit of Nunavik: possible influences of sex and age, British Journal of Nutrition, 102(5): 766-776.

COUNIL, Émilie, Marie-Josée GAUTHIER et Eric DEWAILLY

2011 Alimentation et santé publique dans les communautés Inuits du Nord-du-Québec. Vers un changement de paradigme, in J.-G. Petit, B. Viger, V. Aatami, A. Isherhoff, (dir.), Les Inuits et les Cris du Nord du Québec, Québec, Presses de l'Université du Québec et Rennes, Presses universitaires de Rennes: 237-253.

DAMMAN, Siri, Wenche Barth EIDE et Harriet V. KUHNLEIN

2008 Indigenous peoples' nutrition transition in a right to food perspective, Food Policy, 33(2): 135-155.

DESMARAIS, Annette Aurélie et Hannah WITTMAN

2014 Farmers, Foodies and First Nations: Getting to Food Sovereignty in Canada, The Journal of Peasant Studies, 41(16) : 1153-1173.

DINKOVA-KOSTOVA, Albena T. et Rumen V. KOSTOV

2012 Glucosinolates and isothiocyanates in health and disease, Trends in Molecular Medicine, 18(6) : 337-347. 
DUCHEMIN, Éric, Fabien WEGMULLER et Anne-Marie LEGAULT

2010 Agriculture urbaine: un outil multidimensionnel pour le développement des quartiers, VertigO-la revue électronique en sciences de l'environnement, 10(2).

FAO (FOOD AND AGRICULTURE ORGANIZATION OF THE UNITED NATIONs)

2006 Food Security, Policy Brief, 2.

FLICKER, Sarah, Beth SAVAN, Mary MCGRATH, Brian KOLENDA et Matto MILDENBERGER

2008 "If you could change one thing..." What community-based researchers wish they could have done differently, Community Development Journal, 43(2): 239-253.

FORD, James D.

2009 Vulnerability of Inuit food systems to food insecurity as a consequence of climate change: a case study from Igloolik, Nunavut, Regional Environmental Change, 9(2): 83-100.

FSC (FOOD SECURE CANADA)

2011 Resetting the Table: A People's Food Policy for Canada, Montréal, Food Secure Canada (en ligne, www.peoplesfoodpolicy.ca).

FURGAL, Christopher et Jacinthe SÉGUIN

2006 Climate change, health, and vulnerability in Canadian northern Aboriginal communities, Environmental health perspectives, 114(12): 1964-1970.

HAMM, Michael W. et Anne C. BELLOWS

2003 Community food security and nutrition educators, Journal of Nutrition Education and Behavior, 35(1): 37-43.

HOLZMAN, Sara

2011 Community agriculture in Nunavut: How to ensure successful community greenhouses, mémoire de maîtrise, University of Guelph, Guelph.

HOWES, Melanie-Jayne R. et Monique S.J. SIMMONDS

2014 The role of phytochemicals as micronutrients in health and disease, Current Opinion in Clinical Nutrition and Metabolic Care, 17(6) : 558-566.

HUET, Catherine, Renata ROSOL et Grace M. EGELAND

2012 The prevalence of food insecurity is high and the diet quality poor in Inuit communities, The Journal of Nutrition, 142(3): 541-547.

ISRAEL, Barbara A., Amy J. SCHULZ, Edith A. PARKER et Adam B. BECKER

2001 Community-based Participatory Research: Policy Recommendations for Promoting a Partnership Approach in Health Research, Education for Health, 14(2): 182-197.

KAPUSTA-DUCH, Joanna, Aneta KOPEĆ, Ewa PIATKOWSKA, Barbara BORCZAK et Teresa LESZCZYŃSKA

2012 The beneficial effects of Brassica vegetables on human health, Rocz Panstw Zakl Hig, 63(4): 389-395.

KUHNLEIN, Harriet V., Olivier RECEVEUR, Rula SOUEIDA et Grace M. EGELAND

2004 Arctic indigenous peoples experience the nutrition transition with changing dietary patterns and obesity, The Journal of Nutrition, 134(6): 1447-1453. 
LAMBDEN, Jill, Olivier RECEVEUR, Joan MARSHALL et Harriet V. KUHNLEIN

2006 Traditional and market food access in the Arctic is affected by economic factors, International Journal of Circumpolar Health, 65(4): 331-340.

LORING, Philip A. et Graig S. GERLACH

2010 Outpost gardening in interior Alaska: Food system innovation and the Alaska native gardens of the 1930s through the 1970s, Ethnohistory, 57(2): 183-199.

MADELEY, John

2002 Le commerce de la faim. La sécurité alimentaire sacrifiée sur l'autel du libre-échange, Montréal, Éditions Écosociété, collection Enjeux planète.

NAHO (NATIONAL ABORIGINAL HEALTH ORGANIZATION)

2008 Resource Extraction and Aboriginal Communities in Northern Canada: Gender Considerations, Ottawa, National Aboriginal Health Organization.

ONU (ORGANISATION DES NATIONS UNIES)

1975 Report of the World Food Conference, Rome, 5-16 November 1974, Genève, Organisation des Nations unies (en ligne, http://www.un.org/en/development/ devagenda/food.shtml).

PEARCE, Tristan, James FORD, Ashlee Cunsolo WILLOX et Barry SMITH

2015 Inuit Traditional Ecological Knowledge (TEK), Subsistence Hunting and Adaptation to Climate Change in the Canadian Arctic, Arctic, 68(2): 233-245.

PLAN NUNAVIK

2014 Plan Nunavik, Kuujjuaq, Kativik Regional Government and Makivik Corporation.

POWELL, Bronwen, Patrick MAUNDU, Harriet V. KUHNLEIN et Timothy JOHNS

2013 Wild foods from farm and forest in the East Usambara Mountains, Tanzania, Ecology of food and nutrition, 52(6): 451-478.

POWER, Elaine M.

2008 Conceptualizing food security for Aboriginal people in Canada, Canadian Journal of Public Health/Revue canadienne de santé publique, 99(2): 95-97.

RASTOIN, Jean-Louis et Gérard GHERSI

2010 Le système alimentaire mondial. Concepts et méthodes, analyses et dynamiques, Versailles, Éditions Quae, collection Synthèses.

READING, Charlotte Loppie et Fred WIEN

2009 Health inequalities and the social determinants of Aboriginal peoples' health, Prince George, National Collaborating Centre for Aboriginal Health.

RENTING, Henk, Terry K. MARSDEN et Jo BANKS

2003 Understanding alternative food networks : exploring the role of short food supply chains in rural development, Environment and Planning A, 35(3): 393-411.

RRSSSN (RÉGIE RÉGIONALE DE LA SANTÉ ET DES SERVICES SOCIAUX DU NUNAVIK)

n.d. Guide alimentaire du Nunavik, Kuujjuaq, Régie régionale de la Santé et des Services Sociaux du Nunavik. 
RUIZ-CASTELL, Maria, Gina MUCKLE, Éric DEWAILLY, Joseph L. JACOBSON, Sandra W. JACOBSON, Pierre AYOTTE et Mylène RIVA

2015 Household crowding and food insecurity among Inuit families with school-aged children in the Canadian Arctic, American Journal of Public Health, 105(3): 122-132.

SHARMA, Sangita, Xia CAO, Cindy ROACHE, Annie BUCHAN, Rhonda REID et Joel GITTELSHON

2010 Assessing dietary intake in a population undergoing a rapid transition in diet and lifestyle: the Arctic Inuit in Nunavut, Canada, British Journal of Nutrition, 103(5): 749-759.

\section{SOCIÉTÉ DU PLAN NORD}

2014 Élaborer et mettre en place un modèle bioalimentaire nordique durable en misant sur le potentiel du territoire (en ligne, http://plannord.gouv.qc.ca/fr/priorites-daction/ mise-en-valeur-du-potentiel-economique-du-nord/bioalimentaire/).

SOUCI, Siegfried, Wielfried FACHMANN et Heinrich KRAUT

2008 La composition des aliments. Tableaux des valeurs nutritives, Boca Raton, CRC Press et Stuttgart, MedPharm Scientific Publishers, Wissenschaftliche Verlagsgesellschaft.

SPERLING, Laurence S. et John R. NELSON

2016 History and future of omega-3 fatty acids in cardiovascular disease, Current Medical Research Opinion, 32(2): 301-311.

\section{STATISTIQUE CANADA}

2014 Profil du recensement de Kangiqsujuaq, Ottawa, Statistique Canada.

TARASUK, Valérie

2001 Discussion paper on household and individual food insecurity, document préparé pour Santé Canada (en ligne, http://www.hc-sc.gc.ca/fnan/alt_formats/hpfb-dgpsa/ pdf/nutrition/food_sec_entire-sec_aliments_entier-eng.pdf).

TAYLOR, Juanita

2014 Kuujjuaq hunters and trappers build chicken coop, CBC News, 20 août (en ligne, http://www.cbc.ca/news/canada/north/kuujjuaq-hunters-and-trappers-build-chicken -coop-1.2741457).

WAKEFIELD, Sarah, Kaylen R. FREDRICKSON et Tim BROWN

2015 Food security and health in Canada: Imaginaries, exclusions and possibilities, The Canadian Geographer/Le Géographe canadien, 59(1): 82-92.

WALLERSTEIN, Nina B. et Bonnie DURAN

2006 Using Community-Based Participatory Research to Address Health Disparities, Health Promotion Practice, 7(3) : 312-323.

WU, Ting, Jia FU, Yue-Xin YANG, Li-Shi ZHANG L et Jun-Hua HAN

2009 The effects of phytosterols/stanols on blood lipid profiles: A systematic review with meta-analysis, Asia Pacific Journal of Clinical Nutrition, 18(2): 179-186. 